\title{
Wind induced torsional loads on structures
}

\section{A. Kareem}

Department of Civil Engineering, University of Houston, Texas, USA (Received May 1980; revised July 1980)

For structural reasons, and due to the human biodynamical sensitivity to angular motion, torsional response is an important factor in building design. A torsional motion in a building results from a possible aerodynamic and/or inertial coupling in various degrees of freedom. If the resultant wind forces do not coincide with the centre of mass at each floor level, an eccentric loading pattern can be expected which is responsible for exciting the torsional mode of vibration. An amplification of the response is possible by dynamic inertial coupling, if the vertical elastic axis of the building and the centre of gravity are not in alignment. The torsional response is also sensitive to the ratio of torsional to translational frequencies.

There is no existing wind load description which adequately represents the aerodynamic loading mechanism for torsional response. A number of investigators ${ }^{1,2}$ have used a quasi-steady approach to develop models for the torsional loads, thereby ignoring the important unsteady wake excitation.

The wind-structure interaction problem has so far proved to be intractable and mathematical models based on experimental measurements have been introduced ${ }^{3,4}$ in lieu of the solution of equations for fluid motion around a bluff body. In this report an outline for the development of torsional loads on a rectangular cross-section building has

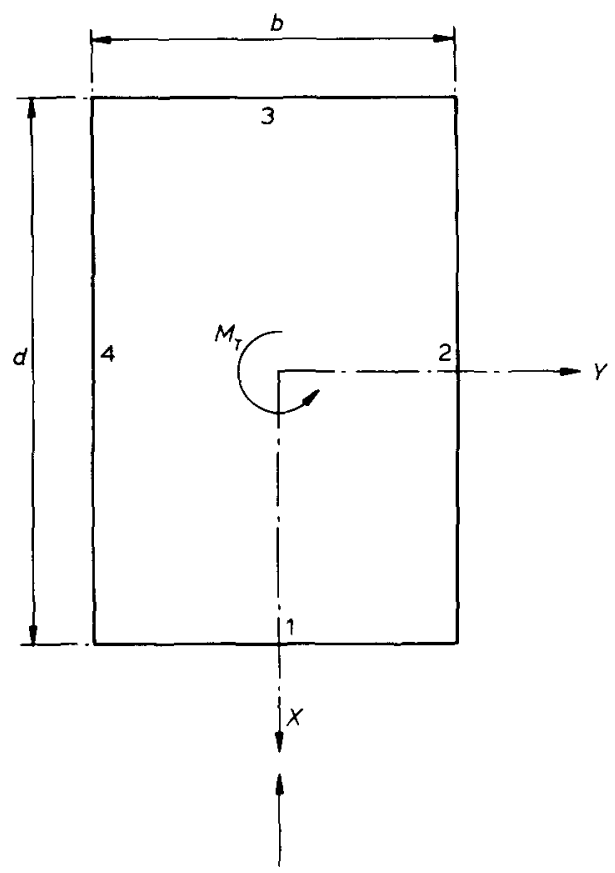

Figure 1 Coordinate system and building plan been presented in frequency domain. Through the use of the spatio-temporal characteristics of pressure fluctuations measured on a scale model in a wind tunnel, expressions for the torsional forcing function can be developed based on numerical integration. A similar technique was successfully used $^{3}$ for the multi-level crosswind loading on a square cross-section building. Reinhold et al. ${ }^{4}$ instrumented a building model with a large number of pressure transducers and used analog circuits to determine fluctuating wind loads. The technique of digital manipulation of data presented here may require fewer transducers with an optimized number of configurations to describe the fluctuating pressure field completely. ${ }^{3}$

A building scale model can be instrumented at a particular level on all the four faces to establish the correlation between various locations on each face (chordwise). In order to extend the loading model to a multi-level forcing function, pressure correlations along the model height (spanwise) are also required. The effective area around each pressure tap and its eccentricity from the building centre can be computed for the evaluation of effective force and associated torsion about the building centre.

The contribution of face 1 (Figure 1) to the torsional loading in spectral representation is given by:

$$
\begin{aligned}
M_{1}(n b / v)= & \int_{-b / 2}^{b / 2} \int_{-b / 2}^{b / 2} \int_{0}^{H} \int_{0}^{H} C O\left(y_{p}, z_{p} y_{q}, z_{q}, \frac{n b}{v}\right) \\
& \times \sqrt{S\left(y_{p}, z_{p}, \frac{n b}{v}\right)} \\
& \times \sqrt{S\left(y_{q}, z_{q}, \frac{n b}{v}\right)} y_{p} y_{q} \mathrm{~d} z_{p} \mathrm{~d} z_{q} \mathrm{~d} y_{p} \mathrm{~d} y_{q}
\end{aligned}
$$

where:

$$
\begin{aligned}
& M_{1}(n b / v)=\text { contribution of face } 1 \\
& C O\left(y_{p}, z_{p}, y_{q}, z_{q}, n b / v\right)=\text { normalized co-spectrum of } \\
& \quad \text { pressure between two arbitrary points } p \text { and } q \text { on face } 1 \\
& S\left(y_{p}, z_{p}, n b / v\right)=\text { power spectral density (PSD) function of } \\
& \quad \text { pressure at location }\left(y_{p}, z_{p}\right) \\
& S\left(y_{q}, z_{q}, n b / v\right)=\text { PSD function at location }\left(y_{q}, z_{q}\right) \\
& n=\text { frequency } \\
& b, d=\text { building dimensions } \\
& v=\text { mean velocity at building height }
\end{aligned}
$$


$H=$ building height

$z=$ height above ground level

The expressions for the contribution of faces 2,3 and 4 can be similarly formulated. The contribution to torsional force from inter-face correlation can be expressed as:

$$
\begin{aligned}
M_{12}(n b / v)= & \int_{-b / 2}^{b / 2} \int_{-d / 2}^{d / 2} \int_{0}^{H} \int_{0}^{H} C O\left(y_{p}, z_{p}, x_{q}, z_{q}, \frac{n b}{v}\right) \\
& \times \sqrt{S\left(y_{p}, z_{p}, \frac{n b}{v}\right)} \\
& \times \sqrt{S\left(x_{q}, z_{q}, \frac{n b}{v}\right)} y_{p} x_{q} \mathrm{~d} z_{p} \mathrm{~d} z_{q} \mathrm{~d} x_{q} \mathrm{~d} y_{p}
\end{aligned}
$$

where:

$M_{12}(n b / v)=$ contribution of torsional force due to correlation of pressure on faces 1 and 2

$C O\left(y_{p}, z_{p}, x_{q}, z_{q}, n b / v\right)=$ normalized co-spectrum of pressure between two arbitrary points $p$ and $q$ on faces 1 and 2 respectively

$S\left(y_{p}, z_{p}, n b / v\right)=$ PSD of pressure fluctuations at a point $p$ on face 1

$S\left(x_{q}, z_{q}, n b / v\right)=$ PSD of pressure fluctuations at a point $q$ on face 2

The contributions of $M_{13}(n b / v), M_{14}(n b / v), M_{23}(n b / v)$, $M_{24}(n b / v)$, and $M_{34}(n b / v)$ can be formulated on a similar basis.

The power spectral density of torsional force $M_{T}$ is then given by the sum of contributions on each face and their interface components. This is obtained by synthesizing multiple random inputs resulting from faces $1,2,3$ and 4 :

$$
M_{T}\left(\frac{n b}{v}\right)=\sum_{i=1}^{4} M_{i}\left(\frac{n b}{v}\right)+2 \sum_{i=1}^{3} \sum_{j=1+i}^{4} M_{i j}\left(\frac{n b}{v}\right)
$$

The above equation can be written in a discrete matrix form to be compatible with experimental measurements:

$$
\begin{aligned}
M_{T}\left(\frac{n b}{v}\right)= & \sum_{j=1}^{4}\left\{A_{i} L_{i}\right\}_{j}^{T}\left[\sqrt{\left.S_{i}\left(\frac{n b}{v}\right)\right]_{j}^{T}\left[C O\left(\frac{n b}{v}\right)\right]_{j}}\right. \\
& \times\left[\sqrt{\left.S_{i}\left(\frac{n b}{v}\right)\right]_{j}\left\{A_{i} L_{i}\right\}_{j}}\right. \\
& +2 \sum_{j=1}^{3} \sum_{k=j+1}^{4}\left\{A_{i} L_{i}\right\}_{j}^{T}\left[\sqrt{S_{i}\left(\frac{n b}{v}\right)}\right]_{j}^{T} \\
& \times\left[C O\left(\frac{n b}{v}\right)\right]_{j k}\left[\sqrt{S_{i}\left(\frac{n b}{v}\right)}\right]_{k}\left\{A_{i} L_{i}\right\}_{k}
\end{aligned}
$$

where $\left\{A_{i} L_{i}\right\}$ is the vector of respective areas and their location from the building centre at the $i$ th level, $[C O(n b / v)]_{j}$ and $[C O(n b / v)]_{j k}$ are the co-spectral matrices of intraface and interface pressure fluctuations at the $i$ th level, $\left[\sqrt{S_{i}(n b / v)}\right]$ is the diagonal matrix and ' $T$ ' denotes the transpose.

In order to expand this formulation to a multi-level forcing function it is necessary to develop relationships between various levels on the building. The diagonal terms of the cross power spectral density matrix of multi-level torsion loads can be generated from equation (4). For the off-diagonal terms representing inter-level relationship the following equation is used:

$$
\begin{aligned}
M_{T}\left(\frac{n b}{v}\right)= & \sum_{j=1}^{4}\left\{A_{i} L_{i}\right\}_{j}^{T}\left[\sqrt{\left.S_{i}\left(\frac{n b}{v}\right)\right]_{j}^{T}\left[C O\left(\frac{n b}{v}\right)\right]_{j}^{i l}}\right. \\
& \times\left[\sqrt{\left.S_{l}\left(\frac{n b}{v}\right)\right]_{j}\left\{A_{l} L_{l}\right\}_{j}}\right. \\
& +2 \sum_{j=1}^{3} \sum_{m=i+1}^{4}\left\{A_{i} L_{i}\right\}_{j}^{T}\left[\sqrt{S_{i}\left(\frac{n b}{v}\right)}\right]_{j}^{T} \\
& \times\left[C O\left(\frac{n b}{v}\right)\right]_{j m}^{i l}\left[\sqrt{S_{l}\left(\frac{n b}{v}\right)}\right]_{m}\left\{A_{l} L_{l}\right\}_{m}
\end{aligned}
$$

where $[C O(n b / v)]_{j}^{i l}$ and $[C O(n b / v)]_{j m}^{i l}$ are the co-spectra matrices of intraface and interface pressure fluctuations between $i$ th and $l$ th levels.

The quad-spectrum of pressure fluctuations has not been included in the above formulation since being an odd function it is eliminated by double integration. ${ }^{3}$

The methodology presented here would provide a complete picture of local pressure fluctuations and their spatiotemporal variations for the cladding design and the final quantification of integral torsional loading for the design of a structural system.

\section{References}

1 Patrickson, C. P. and P. Friedmann. 'A study of the coupled lateral and torsional response of tall buildings to wind loading', UCLA School of Engineering and Applied Sciences Rep. UCLA ENG 76126, December 1976

2 Foutch, D. A. and E. Safak. 'Torsional vibration of wind-excited structures', Third ASCE/Eng. Mech. Div. Specialty Conf. Austin, Texas, 17-19 September 1979

3 Kareem, A. 'Wind excited motion of buildings', PhD Thesis, Colorado State University, 1978

4 Reinhold, T. A. et al. "The effect of wind direction on the static and dynamic wind loads on a square-section tall building', Proc. 3rd Coll. Industrial Aerodyn., Aachen, Germany, 14-16 June 1978 